soil concentration had no adverse effect on the efficacy of ortho-phthalaldehyde solution and that the sporicidal activity of or tho-phthalaldehyde increased with increasing temperature.

FROM: Chan-Myers H, Roberts C. Effect of temperature and organic soil concentration on biocidal activity of ortho-phthalaldehyde solution. Presented at the Association for Professionals in Infection Control and Epidemiology, Inc, 27th Annual Conference and International Meeting; June 18-22, 2000; Minneapolis, MN.

\section{Sterilization of Bone Allografts}

Coronado and colleagues from the College of Veterinary Medicine, Michigan State University, East Lansing, conducted a study to compare virucidal effects and bone incorporation properties of cortical bone allografts transplanted into specific-pathogen-free (SPF) cats. Allografts consisted of untreated bone from an SPF cat (negative-control group) and bone from 5 feline leukemia virus ( $\mathrm{FeLV}$ )-infected cats that was subjected to sterilization with ethylene oxide (ETO), preservation with glycerol, or no treatment (positive-control group). Bones from the aforementioned groups and 20 8-weekold SPF cats (5 cats/group)were implanted with an allograft from one of the aforementioned groups. After implantation, blood samples were collected weekly to monitor FeLV p27 antigen and antibody titers. Quantification of FeLV provirus was performed on blood samples at weeks 0,4 , and 8 , and donor bone samples at time of implantation. Cats were sacrificed 8 weeks after transplantation, and graft sites were evaluated.

All results for negative-control cats were negative. All ETO-group cats had negative results for antigen and provirus in blood, whereas one cat had a low antibody titer. Although three ETO-treated allografts were positive for provirus, the DNA appeared denatured. One cat in the glycerol group had positive results for all tests in blood samples. All glycerol-preserved allografts were positive when tested for provirus. All results for positive-controlgroup cats were positive. Differences in incorporation of bone grafts were not observed.

It was concluded that glycerol preservation of FeLVinfected bone allografts did not eliminate transmission of retrovirus to recipients. In contrast, ETO sterilization appeared to denature DNA and prevent infection. Treatments did not affect incorporation of bone grafts in young cats. This research may have implications for proper sterilization of bone grafts in humans.

FROM: Coronado GS Jr, Swenson CL, Martinez SA, Burkhardt KS, Arnoczky SP. Effects of a 98\% solution of glycerol or sterilization with ethylene oxide on FeLV in bone allografts and effects on bone incorporation of allografts in cats. Am J Vet Res 2000;61:665-671.

\section{Infection Risks in Pediatric Organ Transplantation}

Infectious complications are a major cause of morbidity and mortality after organ transplantation. There are several reports on infections during the first months after transplantation, but there are very few data regarding infections in long-term survivors of pediatric organ transplantation. Their and colleagues from the Hospital for Children and Adolescents, University of Helsinki, Finland, retrospectively analyzed the incidence and type of infections in 56 children who underwent 59 liver or renal transplantations. Follow-up was begun when the patient was sent home after a successful operation. All of the children received triple immunosuppression.

During a mean follow-up of 4.8 years (total, 286 patient-years), 1,540 episodes of infection were recorded. The median incidence was 4.8 episodes per patient-year. The greatest number was seen in the smallest children, 3 to 6 months after transplantation. Viral upper respiratory tract infections were the most common problem, accounting for one half of the episodes (2.7 episodes/patient-year). Gastroenteritis was the second most common viral infection. Only 45 episodes of infection with herpesviruses were recorded, and 7 of those were caused by cytomegalovirus. Otitis media and sinusitis were the most common bacterial infections and complicated upper respiratory infection in $23 \%$ of episodes. Thirty-nine episodes of urinary tract infections were diagnosed, 31 in children with renal transplants. Other bacterial infections were rare, and only three episodes of verified bacterial sepsis were diagnosed.

The authors concluded that the frequency and type of infections in children with liver and renal transplants who are on triple immunosuppression are quite similar to those in age-matched healthy children.

FROM: Their M, Holmberg C, Lautenschlager I, Hockerstedt K, Jalanko H. Infections in pediatric kidney and liver transplant patients after perioperative hospitalization. Transplantation 2000;69:1617-1623.

\section{Expiration of Multidose Vials of PPD}

The United States Pharmacopeial Convention (USP) recently issued an alert regarding the expiration dating of tuberculin purified protein derivative (Aplisol, Parkedale Pharmaceuticals, Rochester, MI) diluted after the vial is entered. Pharmacists should be aware that, once entered, vials should be used for no longer than 30 days. This information is mentioned in the package insert for Aplisol, but not on the box or the vial label. Product quality concerns can be reported to the USP on line at www.usp.org or by calling 800-487-7776.

FROM: US Pharmacopeial Practitioner reporting at www.usp.org. 